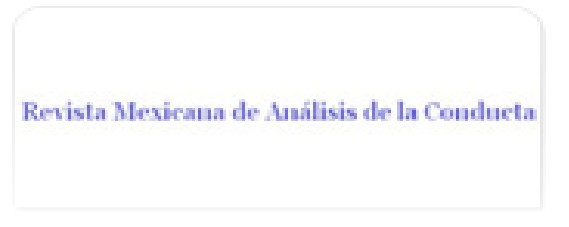

Revista Mexicana de Análisis de la Conducta ISSN: 0185-4534

editora@rmac-mx.org

Sociedad Mexicana de Análisis de la Conducta México

MATEOS MORFÍN, REBECA; FLORES AGUIRRE, CARLOS EFECTOS DEL INTERVALO ENTRE CICLOS Y CONTROL DEL ESTÍMULO EN PROGRAMAS DEFINIDOS TEMPORALMENTE

Revista Mexicana de Análisis de la Conducta, vol. 35, septiembre, 2009, pp. 101-116 Sociedad Mexicana de Análisis de la Conducta Guadalajara, México

Disponible en: http://www.redalyc.org/articulo.oa?id=59312304008

Cómo citar el artículo

- Número completo

- Más información del artículo

- Página de la revista en redalyc.org

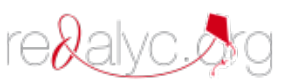

Sistema de Información Científica Red de Revistas Científicas de América Latina, el Caribe, España y Portugal Proyecto académico sin fines de lucro, desarrollado bajo la iniciativa de acceso abierto 


\title{
EFECTOS DEL INTERVALO ENTRE CICLOS Y CONTROL DEL ESTÍMULO EN PROGRAMAS DEFINIDOS TEMPORALMENTE
}

\author{
INTER-CYCLE INTERVAL EFFECTS AND STIMULUS CONTROL IN \\ TEMPORALLY DEFINED SCHEDULES
}

\author{
REBECA MATEOS MORFÍN Y CARLOS FLORES AGUIRRE ${ }^{1}$ \\ UNIVERSIDAD DE GUADALAJARA \\ CENTRO DE ESTUDIOS E INVESTIGACIONES EN COMPORTAMIENTO
}

\begin{abstract}
RESUMEN
Dos grupos de ratas fueron expuestos a programas definidos temporalmente $\left(t^{\mathrm{D}}=30 \mathrm{~s}, \mathrm{t}^{\Delta}=30 \mathrm{~s}\right)$ que difirieron entre sí por la duración de un intervalo entre ciclos ( $3 \circ 30 \mathrm{~s}$ ) introducido entre el final de $t^{\Delta}$ y el inicio de $t^{D}$. Para evaluar el desarrollo de funciones de estímulo, $t^{D}$ y $t^{\Delta}$ se correlacionaron con señales particulares. La tasa de respuesta fue más alta en $t^{D}$ que en $t^{\Delta}$ para el grupo expuesto al intervalo entre ciclos más largo, mientras que para el otro grupo se observó el efecto inverso. Los resultados se discuten en términos del desarrollo de funciones de estímulo, resaltando una posible función de reforzador condicionado de la señal correlacionada con el subciclo $t^{\Delta}$.
\end{abstract}

Palabras clave: programas definidos temporalmente, tasa de respuesta, intervalo entre ciclos, ratas, funciones de estímulo.

1. La correspondencia sobre este artículo deberá enviarse a cualquiera de los autores a: Universidad de Guadalajara, Centro de Estudios e Investigaciones en Comportamiento. Francisco de Quevedo 180, Col. Arcos Vallarta, Guadalajara, Jalisco. México, CP 44130, o a los correos electrónicos: rebecamateos@gmail.com ó carlos.flores@cucba.udg.mx. Una versión preliminar de este trabajo se presentó en el xVIII Congreso de la Sociedad Mexicana de Análisis de la Conducta. Xalapa, Veracruz, México, noviembre de 2007.

Recibido: 23 de marzo de 2009. Revisado: 15 de mayo de 2009. Aceptado: 15 de julio de 2009. 


\begin{abstract}
Two groups of rats were exposed to temporally defined schedules $\left(\mathrm{t}^{\mathrm{D}}=30\right.$ $\mathrm{s}, \mathrm{t}^{\Delta}=30 \mathrm{~s}$ ) that differed by the length of an inter cycle interval ( 3 or $30 \mathrm{~s}$ ) intruded between the end of $t^{\Delta}$ and the beginning of $t^{D}$. In order to evaluate stimulus functions development, both $\mathrm{t}^{\mathrm{D}}$ and $\mathrm{t}^{\Delta}$ were correlated with particular cues. Response rate was higher in $t^{D}$ than in $t^{\Delta}$ for the group exposed to the longer inter-cycle interval, whereas for the other group the inverse effect was observed. Results are discussed in terms of stimulus functions development, highlighting a possible conditioned reinforcement function of the cue correlated with $\mathrm{t}^{\Delta}$ subcycle.
\end{abstract}

Keywords: Temporally defined schedules, response rates, interval between cycles, rats, stimulus functions.

Un hallazgo que ha sido sistemático y contradictorio a los resultados reportados en la literatura del control del estímulo (e g. Hearst, Besley \& Farthing, 1970; Mackintosh, 1977; Rilling, 1977; Terrace, 1966) empleando programas de reforzamiento (Ferster \& Skinner, 1957), es que cuando se emplean programas definidos temporalmente (Schoenfeld \& Cole, 1972) se observa una frecuencia de respuesta más alta durante el estímulo correlacionado con el no reforzamiento de la respuesta que durante el periodo en el cual la respuesta es reforzada. Este resultado ha sido reportado consistentemente en una serie de trabajos (e.g. Ribes, Mayoral, Torres \& Ibáñez, 2000; Ribes \& Torres, 1996, 1997; Ribes, Torres \& Mayoral, 2000a, b; Ribes, Torres \& Mayoral, 2002; Ribes, Torres \& Piña, 1999).

Los programas definidos temporalmente consisten en un ciclo repetitivo de tiempo (ciclo $\mathrm{T}$ ) dividido en dos subciclos o periodos que alternan, uno de ellos denominado tiempo discriminativo $\left(t^{D}\right)$ y el otro denominado tiempo delta $\left(t^{\Delta}\right)$. La primera respuesta que ocurre en $t^{D}$ es reforzada, mientras que las respuestas emitidas en $t^{\Delta}$ normalmente no son reforzadas o se refuerzan con una menor probabilidad que en $\mathrm{t}^{\mathrm{D}}$ (Schoenfeld \& Cole, 1972).

Los resultados reportados por Ribes et al. (1999; 2000; 2000a; 200b; 2002) y por Ribes y Torres $(1996 ; 1997)$ se han interpretado atendiendo a las características de los programas temporales en contraste con las de los programas de reforzamiento tipo Ferster y Skinner (1957). Una característica de los programas definidos temporalmente es que de no ocurrir respuesta durante $t^{D}$ el reforzador programado para ese ciclo se pierde, mientras que en los programas de reforzamiento tradicionales no existe la posibilidad de pérdida de reforzadores (Schoenfeld \& Cole, 1972; Schoenfeld, Cumming \& Hearst, 1956). Una característica adicional de los procedimientos empleados 
en la serie de estudios reportados por Ribes et al. y por Ribes y Torres, es que el estímulo correlacionado a $t^{D}$ se cancela con la primera respuesta que ocurre en su presencia, mientras que la señal correlacionada a $t^{\Delta}$ no se ve modificada por la ocurrencia de respuestas.

En un trabajo reciente, Flores, Mateos, Villanueva y Ortiz (2007) evaluaron los efectos del mantenimiento y cancelación de las señales correlacionadas a $t^{D}$ y $t^{\Delta}$ sobre la tasa de respuesta. Expusieron a palomas a un ciclo $T$ de $60 \mathrm{~s}$, en el que cada subciclo o periodo tuvo una duración de $30 \mathrm{~s}$. Para un grupo de sujetos una luz de color verde, correlacionada al periodo $t^{\mathrm{D}}$, se cancelaba con la emisión de la primera respuesta, mientras que una luz de color rojo, correlacionada a $t^{\Delta}$, era coextensiva a dicho subciclo independientemente de que ocurriesen o no respuestas en su presencia. Para otro grupo de sujetos el estímulo correlacionado a $t^{\mathrm{D}}$ era coextensivo al subciclo, mientras que el estímulo correlacionado a $t^{\Delta}$ se cancelaba a la primera respuesta que ocurriera en su presencia.

Flores et al. (2007) reportaron una frecuencia de respuesta más alta en $t^{\Delta}$ que en $t^{D}$. Estos resultados fueron consistentes con los reportados por Ribes et al. (1999; 2000; 2000a; 200b; 2002) y por Ribes y Torres (1996; 1997); en particular, en el grupo en el que la señal correlacionada a $t^{D}$ se cancelaba por la ocurrencia de la primera respuesta y la señal de $t^{\Delta}$ era coextensiva a este subciclo. Flores et al. también reportaron que sus sujetos mostraron un elevado porcentaje de reforzadores obtenidos, a diferencia de lo reportado en la serie de estudios de Ribes et al. y de Ribes y Torres, en los que se ha documentado que las ratas obtienen un escaso porcentaje de reforzadores programados. Dada la discrepancia de resultados en términos del porcentaje de reforzadores obtenidos, la ausencia de control del estímulo reportada en los estudios de Ribes et al. y en los de Ribes y Torres no puede ser explicada por la restricción que imponen los programas temporales para la entrega del reforzador ni por la pérdida de reforzadores.

En un par de trabajos en los que se utilizaron ratas como sujetos, Flores y Mateos (2007) y Peralta, Mateos, Villanueva y Flores (2007) evaluaron los efectos de variar la duración del estímulo correlacionado a $t^{\Delta}$ sobre la frecuencia de respuesta. Flores y Mateos variaron entre fases la duración de $t^{\Delta}$ y de la señal que le era coextensiva $(30,15$ y $3 \mathrm{~s})$. De manera similar a los procedimientos empleados en la serie de estudios reportada por Ribes et al. (1999; 2000; 2000a; 200b; 2002) y por Ribes y Torres (1996; 1997), la primera respuesta emitida en el período $t^{\mathrm{D}}$ cancelaba la luz correlacionada y entregaba una gota de agua, mientras que la luz correlacionada a $t^{\Delta}$ no se cancelaba por la emisión de respuestas. De manera similar al procedimiento descrito en el estudio anterior, Peralta et al. evaluaron los efectos de la duración de $t^{\Delta}$, excepto que en este estudio la señal correlacionada a $t^{D}$ no se cancelaba por la emisión de la respuesta. En ambos estudios se encontró una mayor 
frecuencia de respuesta durante la señal correlacionada al no reforzamiento que durante $t^{\mathrm{D}}$. Asimismo, reportaron que la reducción en la duración de $t^{\Delta}$ resultó en incrementos progresivos en la frecuencia de respuesta durante dicho periodo, es decir, mayores frecuencias de respuesta en la condición con $t^{\Delta}$ de $3 \mathrm{~s}$ que durante la condición con una duración de $t^{\Delta}$ igual a $30 \mathrm{~s}$.

Los resultados anteriores fueron interpretados en términos del posible desarrollo de una función reforzante por parte del estímulo coextensivo al periodo $t^{\Delta}$ dada su proximidad temporal con el siguiente ciclo, y en consecuencia con la señal correlacionada al período $t^{\mathrm{D}}$ en cuya presencia la primera respuesta procuró el reforzador. Esta interpretación se puede ver fortalecida dado que existen trabajos en los que se ha reportado que el intervalo estímulo-reforzador controla la tasa de respuesta. Por ejemplo, Bersh (1951, Experimento 1) evaluó la fuerza que desarrolla un estímulo como reforzador condicionado en función de la duración del intervalo estímulo-reforzador. Este autor expuso a ratas a diferentes duraciones del intervalo estímulo-reforzador $(0,0.5,1,2,4$ y $10 \mathrm{~s})$ y reportó que el número de respuestas de presión a la palanca decrementó en función de alargar el intervalo estímulo-reforzador. Este resultado es consistente con el observado en los estudios de Flores y Mateos (2007) y Peralta et al. (2007), quienes encontraron una tasa de respuesta más alta ante el estímulo correlacionado a una duración corta de $t^{\Delta}$ (3 s) comparada con una mayor duración de $t^{\Delta}(30 \mathrm{~s})$.

En otro estudio, Farmer y Schoenfeld (1966) evaluaron los efectos de variar la posición temporal de un estímulo durante el intervalo entre reforzadores y reportaron que cuando una luz se ubicaba inmediatamente antes del reforzador (máxima contigüidad), los sujetos respondieron con tasas mucho más elevadas que cuando al luz se ubicaba en una posición más alejada del momento en el que ocurría el reforzador. Farmer y Schoenfeld también reportaron que dependiendo de la posición temporal que guardara el estímulo (luz) en el intervalo entre reforzadores, el estímulo podía desarrollar diferentes funciones (e.g. estímulo discriminativo, reforzador condicionado o de segundo orden).

En un estudio reciente, Escobar y Bruner (2008) utilizando un procedimiento de respuestas de observación, reportaron que la separación temporal entre los componentes de reforzamiento y de extinción, mediante la introducción de un intervalo sin consecuencias, modificó la tasa de respuesta de observación durante el componente de extinción. Específicamente, encontraron que cuando se agregó el intervalo sin consecuencias al final del componente de extinción las tasas de respuesta de observación durante el componente de extinción disminuyeron respecto a una condición de línea base en la que no estaba presente el intervalo, y que cuando el intervalo se introdujo antes de iniciar el componente de extinción las tasas de respuesta de observación no se vieron sustancialmente modificadas con respecto a la 
condición de línea base, en la que no estaba presente el intervalo entre componentes. Con base en estos resultados, Escobar y Bruner concluyeron que el mantenimiento de las respuestas de observación durante el componente de extinción puede ser explicado por la función de reforzador condicionado que desarrolla el estímulo correlacionado al componente de extinción, como resultado de los eventuales apareamientos entre la señal correlacionada al componente de extinción y el reforzador, así como de la contigüidad entre la señal que puede ocurrir en el componente de extinción y el subsecuente componente de reforzamiento.

De manera similar a la manipulación reportada por Escobar y Bruner (2008), una manipulación que resulta simple y podría resultar conducente para explorar la posibilidad del desarrollo de una función reforzante por parte del estímulo correlacionado al periodo $t^{\Delta}$, es alterar la contigüidad entre la señal correlacionada a $t^{\Delta}$ y el inicio del siguiente ciclo $T$ mediante la imposición de un intervalo entre ciclos. Esta manipulación podría mostrar si la señal correlacionada a $t^{\Delta}$ desarrolla una posible función reforzante, pudiendo explicar el hecho de observar frecuencias de respuesta más altas durante la señal correlacionada al subciclo $t^{\Delta}$ que durante el subciclo $t^{\mathrm{D}}$.

Una de las características de los programas definidos temporalmente es su naturaleza continua, es decir, la sucesión de los diferentes ciclos $T$ que conforman una sesión experimental. Si bien la imposición de un intervalo entre ciclos modifica parcialmente la sucesión continua de los ciclos T, introducir dicho intervalo puede ser justificable con el propósito de modificar la contigüidad temporal entre la señal correlacionada a $t^{\Delta}$ y el inicio de $t^{D}$ en el ciclo subsecuente. En este sentido, el presente estudio se diseñó con el propósito de evaluar el efecto de diferentes duraciones del intervalo entre ciclos sobre las tasas de respuesta en $\mathrm{t}^{\mathrm{D}}$ y $\mathrm{t}^{\Delta}$ y la distribución del responder en programas definidos temporalmente.

\section{MÉTODO}

\section{Sujetos}

Se utilizaron seis ratas macho Wistar, de tres meses de edad al inicio del estudio e ingenuas experimentalmente. Los animales se mantuvieron en un régimen de privación de agua por un periodo de $23.5 \mathrm{~h}$ diarias y con acceso libre al alimento en su caja habitación.

\section{Aparatos}

Se utilizaron cuatro cámaras experimentales para ratas marca MED (ENV$008)$, cada caja estuvo equipada con un dispensador de agua (ENV-202M), una palanca y una tecla translúcida de $2.5 \mathrm{~cm}$ de diámetro en la que se pro- 
yectaron luces de diferente color (rojo y verde). La palanca se encontraba a $2.5 \mathrm{~cm}$ del piso y requirió de una fuerza de $0.13 \mathrm{~N}$ para cerrar el microswitch. Como reforzador se utilizó una gota de agua de $0.01 \mathrm{cc}$ que se presentó por medio de la activación del dispensador de agua. Cada cámara experimental se colocó dentro de un cubículo de aislamiento acústico (ENV-022M), provisto con un ventilador que sirvió como ruido blanco y facilitó la circulación del aire al interior de la cámara. La programación, registro y colección de eventos se realizó mediante un equipo de cómputo, una interfase y el software $M E D$ $P C$ IV para ambiente Windows.

\section{Procedimiento}

En todos los sujetos se moldeó la respuesta a la palanca exponiéndolos a un programa concurrente tiempo fijo y reforzamiento continuo (Conc TF 30sRFC). Se utilizó el programa TF para la entrega de agua independiente de la respuesta y favorecer el acercamiento del sujeto al bebedero y propiciar el consumo de la gota de agua; mientras que el programa de reforzamiento continuo procuró el mantenimiento de la respuesta de presión a la palanca. El moldeamiento concluyó una vez que los sujetos obtuvieron 100 gotas de agua conforme a un programa de reforzamiento continuo en cada una de tres sesiones consecutivas.

Concluido el establecimiento de la respuesta se empleó un programa definido temporalmente, consistente en un ciclo $\mathrm{T}$ de $60 \mathrm{~s}$ dividido en periodos $t^{\mathrm{D}}$ y $\mathrm{t}^{\Delta}$ con una duración de $30 \mathrm{~s}$ cada uno. Los periodos siempre se presentaron en el mismo orden iniciando con $t^{D}$. El periodo $t^{D}$ fue iluminado con una luz verde que se canceló a la primera respuesta que ocurría en su presencia, seguido por la entrega de una gota de agua. El periodo $t^{\Delta}$ fue iluminado con una luz roja. Las respuestas que ocurrieron en $t^{\Delta}$ no tuvieron ninguna consecuencia programada, por lo que la luz roja fue coextensiva a dicho subciclo. Para tres ratas (sujetos 1,2 y 3 ) la duración del intervalo entre ciclos (IEC) fue de $3 \mathrm{~s}$ (Grupo IEC-3); para otras tres ratas (sujetos 4, 5 y 6 ) la duración del IEC fue de $30 \mathrm{~s}$. Durante los subciclos y el IEC la cámara experimental permaneció sin iluminación general. Los sujetos fueron expuestos a estas condiciones durante 20 sesiones. El criterio para dar por concluida la exposición al programa estuvo basado en que en otros trabajos, también con ratas y empleando programas temporales con señales correlacionadas a los subciclos $t^{D}$ y $t^{\Delta}$, se han observado resultados consistentes cuando los sujetos han sido expuestos a 10, 20 o mas de 100 sesiones (e.g. Serrano, Moreno, Camacho, Aguilar \& Carpio, 2006; Ribes et al., 1999; 2000; 2000a; 200b; 2002; Ribes \& Torres, 1996; 1997). Cada sesión estuvo conformada por 60 ciclos. Las sesiones experimentales se condujeron durante seis días de la semana. En la figura 1 se muestra una representación de las condiciones experimentales generales y de la separación entre ciclos. 


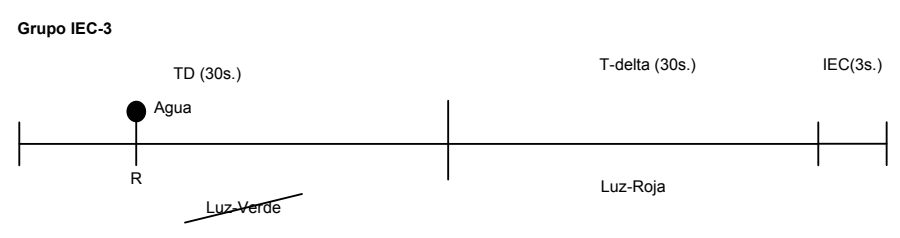

Grupo IEC-30

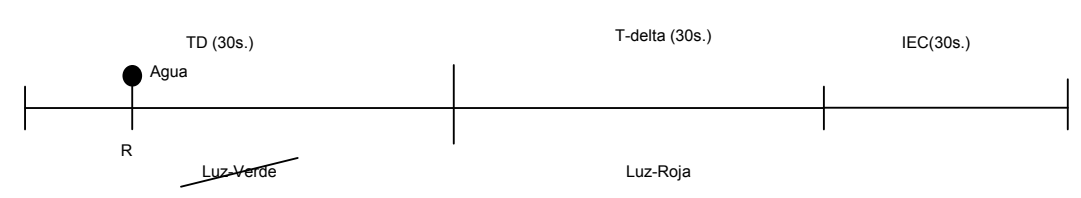

Figura 1. Representación esquemática de la duración de $t^{D}, t^{\Delta}$ y del intervalo entre ciclos para los sujetos del grupo con un IEC de 3 s y los sujetos del grupo con un IEC de $30 \mathrm{~s}$.

\section{Resultados}

Para mostrar la distribución de respuestas durante el intervalo entre reforzadores, se fraccionaron los subciclos $t^{D}$ y $t^{\Delta}$ y el IEC en bloques de $3 \mathrm{~s}$. La figura 2 muestra la distribución de respuestas para los sujeto de cada grupo como una media de las primeras y últimas cinco sesiones. En la parte izquierda de la figura se presentan los datos correspondientes a los sujetos del Grupo con un IEC de $3 \mathrm{~s}$, mientras que en la parte de la derecha se presentan los datos de los sujetos con un IEC de $30 \mathrm{~s}$.

En relación a los sujetos con un IEC de $3 \mathrm{~s}$, se pueden observar diferencias en las distribuciones de respuesta entre las primeras y las últimas cinco sesiones. En particular, en las últimas sesiones se encontró un incremento progresivo en la frecuencia de respuesta desde el inicio del subciclo $t^{\Delta}$, exceptuando al sujeto $\mathrm{S} 1$ para el que se puede observar este efecto desde las primeras sesiones. De manera general se pueden observar distribuciones de respuesta en forma de "U", en las que el responder se concentró en el primero de los subintervalos de $t^{\mathrm{D}}$ y durante el IEC. En lo que respecta a los sujetos del grupo con un IEC de $30 \mathrm{~s}$, se pueden observar distribuciones de respuesta similares entre las primeras y últimas cinco sesiones. En particular, durante las últimas sesiones se encontró que el responder se concentró en los primeros subintervalos de $t^{D}$ y que los sujetos se mantuvieron prácticamente sin responder durante $t^{\Delta}$ y durante el IEC. 
IEC-3

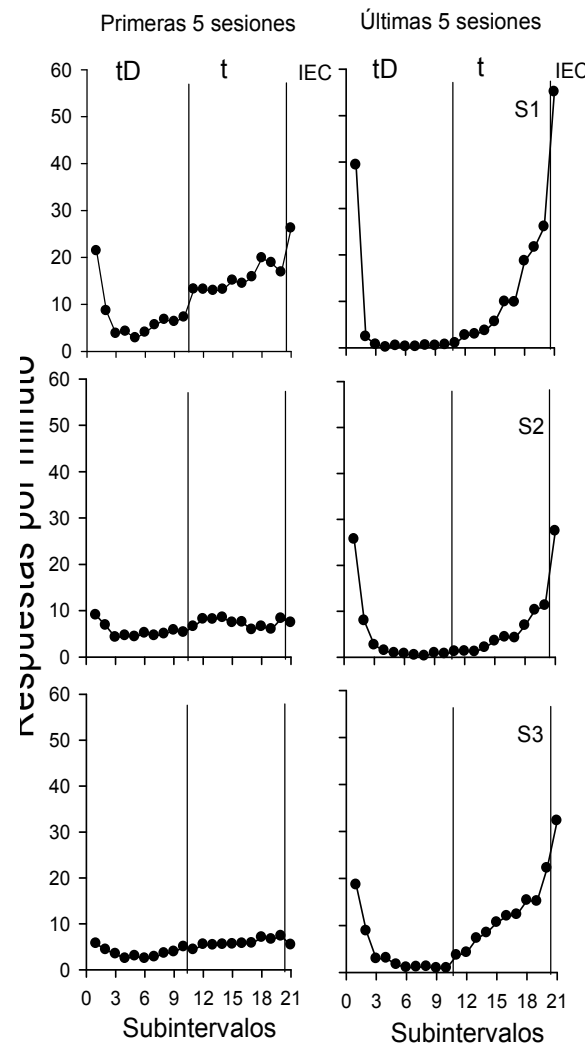

IEC-30

Primeras 5 sesiones Últimas 5 sesiones
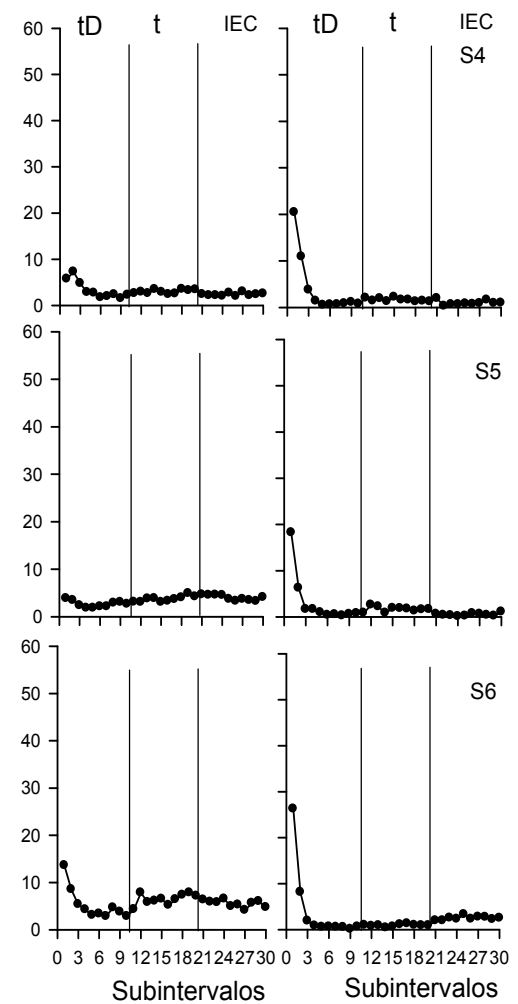

Figura 2. Frecuencia de respuesta de cada sujeto durante cada subintervalo del ciclo $T$ y durante el intervalo entre ciclos. En la parte izquierda se presentan los datos correspondientes a los sujetos del grupo con un IEC de 3 $s$, mientras que en la parte derecha se presentan los datos de los sujetos del grupo con un IEC de $30 \mathrm{~s}$.

En la figura 3 se presentan las tasas de respuesta en $t^{D}$ y en $t^{\Delta}$, promedio de las primeras y últimas cinco sesiones de cada sujeto del grupo con un IEC de $3 \mathrm{~s}$. En esta figura se puede observar que la tasa de respuesta en $\mathrm{t}^{\mathrm{D}}$ fue más baja que la tasa en $t^{\Delta}$ tanto en las primeras como en las últimas cinco sesiones para todos los sujetos, excepto en el sujeto $\mathrm{S} 2$ durante las últimas cinco sesiones, en el que se observa una tasa ligeramente más alta en $t^{\Delta}$ que 
en $\mathrm{t}^{\mathrm{D}}$. Las tasas de respuesta promedio durante las primeras cinco sesiones fueron 5.37 en $\mathrm{t}^{\mathrm{D}}$ y $9.47 \mathrm{en}^{\Delta}$, mientras que durante las últimas cinco sesiones fueron 4.19 en $\mathrm{t}^{\mathrm{D}}$ y 8.59 en $\mathrm{t}^{\Delta}$.

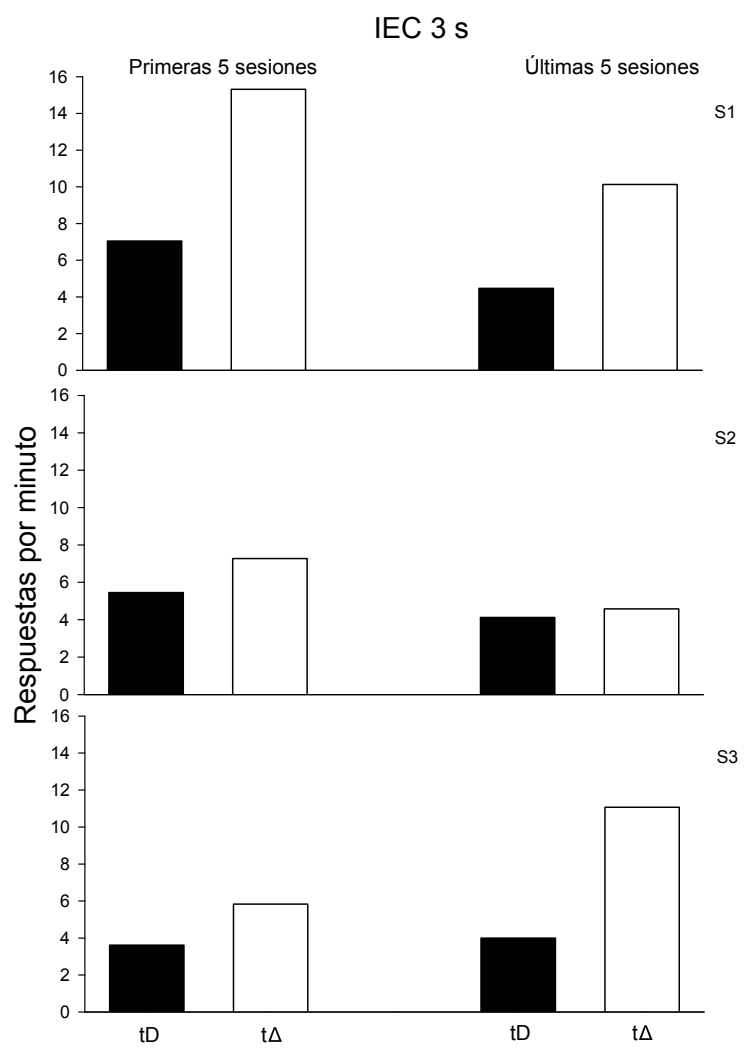

Figura 3. Tasa de respuesta en $t^{D}$ y $t^{\Delta}$ de cada sujeto del grupo con un IEC de $3 \mathrm{~s}$. En la columna de la izquierda se presentan los datos correspondientes a las primeras cinco sesiones, en la columna de la derecha se presentan los datos correspondientes a las últimas cinco sesiones.

En la figura 4 se presentan las tasas de respuesta en $t^{\mathrm{D}}$ y en $\mathrm{t}^{\mathrm{A}}$, promedio de las primeras y últimas cinco sesiones de los sujetos con un IEC de $30 \mathrm{~s}$. En esta figura se observa que durante las primeras cinco sesiones la tasa de respuesta en $t^{\Delta}$ fue más alta que la tasa en $\mathrm{t}^{\mathrm{D}}$, excepto para el sujeto $\mathrm{S} 4$. Du- 
rante las últimas cinco sesiones se puede observar que la tasa de respuesta en $t^{D}$ es más alta que en $t^{\Delta}$ en los tres sujetos. Las tasas de respuesta promedio de los tres sujetos durante las primeras cinco sesiones fueron 3.68 en $t^{D}$ y 4.17 en $t^{\Delta}$, mientras que durante las últimas cinco sesiones fueron 3.70 y 1.34 en $\mathrm{t}^{\mathrm{D}}$ y $\mathrm{t}^{\Delta}$, respectivamente.

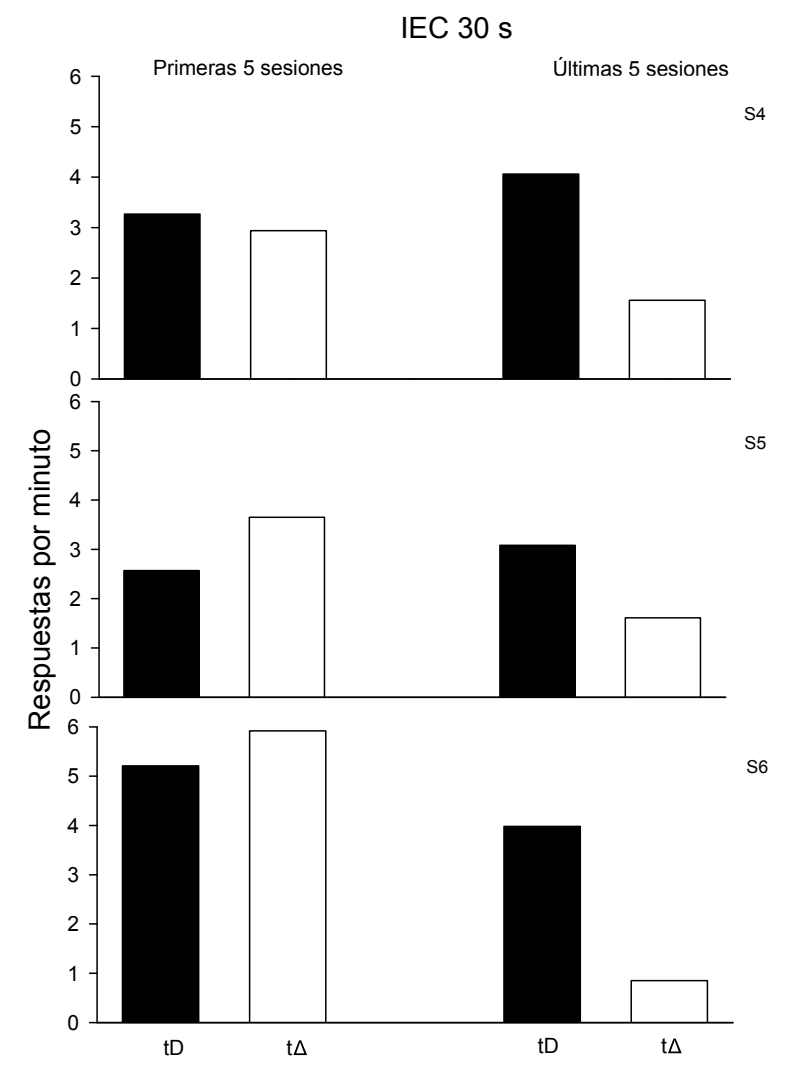

Figura 4. Tasa de respuesta en $t^{D}$ y $t^{\Delta}$ de cada sujeto del grupo con un IEC de $30 \mathrm{~s}$. En la columna de la izquierda se presentan los datos correspondientes a las primeras cinco sesiones, en la columna de la derecha se presentan los datos correspondientes a las últimas cinco sesiones. 
El porcentaje de reforzadores obtenidos ha sido una variable dependiente sistemáticamente reportada en los trabajos que utilizan programas definidos temporalmente y señales diferenciales correlacionadas a cada subciclo. Es por ello que en la figura 5 se presenta el porcentaje de reforzadores obtenidos en cada sesión de los sujetos del Grupo IEC $3 \mathrm{~s}$ (panel superior) y de los sujetos del Grupo IEC 30 s (panel inferior). El porcentaje de reforzadores obtenidos en cada sesión se calculó con base en el total de reforzadores programados en cada sesión (60). En la figura se observa que conforme transcurren las sesiones, tanto los sujetos del Grupo IEC $3 \mathrm{~s}$ como los del Grupo IEC $30 \mathrm{~s}$ muestran una tendencia a obtener un mayor porcentaje de reforzadores. Hacia la parte final del estudio, los porcentajes de reforzadores obtenidos son superiores a $80 \%$ prácticamente en todos los sujetos.

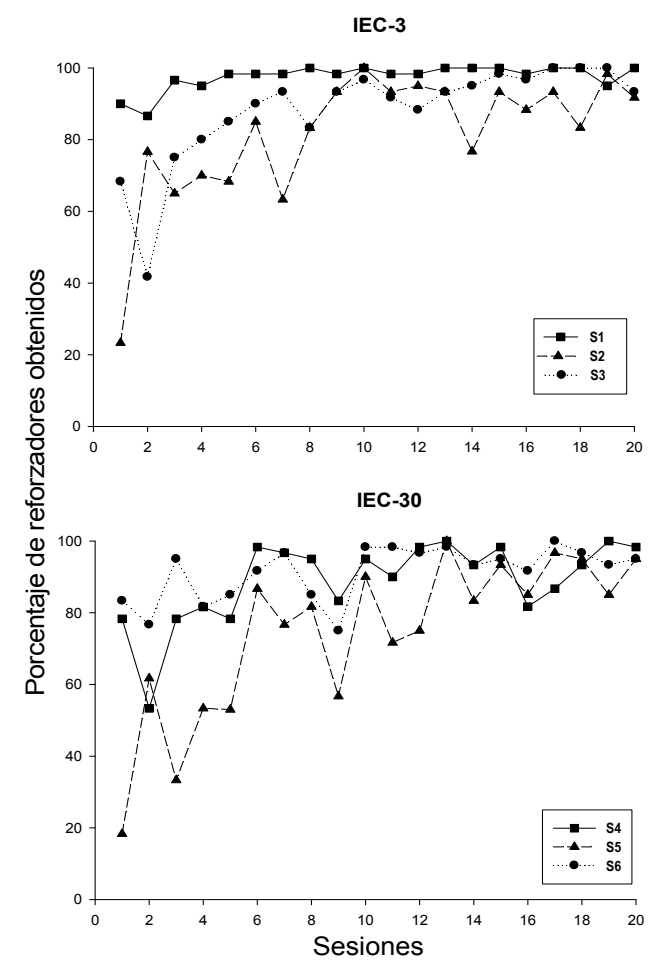

Figura 5. Porcentaje de reforzadores obtenidos por sesión de cada sujeto, panel superior sujetos del Grupo IEC 3 s, panel inferior sujetos del Grupo IEC $30 \mathrm{~s}$. 


\section{DISCUSIÓN}

El presente estudio se diseñó con el propósito de evaluar los efectos de introducir un IEC como una forma de determinar la función que desarrolla el estímulo correlacionado al subciclo $t^{\Delta}$ y su control sobre la tasa y distribución del responder. Como se observó en la sección de resultados, una mayor duración del IEC (30 s) resultó en frecuencias de respuesta más bajas durante el estímulo correlacionado a $t^{\Delta}$ que durante el subciclo $t^{D}$. Este hallazgo no es consistente con algunos trabajos en los que se han reportado mayores frecuencias de respuesta en $t^{\Delta}$ que en $t^{D}$ (e.g. Ribes \& Torres, 1996, 1997; Weissman, 1961, 1965).

Alterar la contigüidad temporal por una mayor separación entre ciclos resultó en diferentes frecuencias de respuesta en $t^{\mathrm{D}}$ y $\mathrm{t}^{\Delta}$. Observar frecuencias de respuesta más altas en $\mathrm{t}^{\Delta}$ que en $\mathrm{t}^{\mathrm{D}}$ en los sujetos con un IEC de $3 \mathrm{~s}$ y mayores frecuencias en $\mathrm{t}^{\mathrm{D}}$ que en $\mathrm{t}^{\Delta}$ en los sujetos con un IEC de $30 \mathrm{~s}$, sugiere que la función desarrollada por el estímulo correlacionado a $t^{\Delta}$ se modificó por la duración del IEC, es decir, por su contigüidad con el siguiente ciclo T y con el reforzador que ocurrió en $\mathrm{t}^{\mathrm{D}}$.

En varios estudios (e.g. Ribes et al. 1999; 2000; 2000a; 200b; 2002; Ribes $\&$ Torres, 1996; 1997) se ha reportado una mayor frecuencia de respuesta durante el estímulo correlacionado con el periodo $t^{\Delta}$, es decir, durante el periodo de extinción. Como se mencionó en la sección introductoria, la ausencia de control del estímulo fue explicada por Ribes et al. y por Ribes y Torres atendiendo a la restricción temporal que caracteriza a este tipo de programas para la procuración del reforzador por la primera respuesta que ocurre durante el subciclo $t^{\mathrm{D}}$.

Dada la limitada capacidad visual que se ha reportado en las ratas (Harrison, 1994), Ribes et al. (2000) evaluaron los efectos de utilizar estímulos auditivos en lugar de luces de diferente color (rojo y verde) correlacionadas a los subciclos $t^{D}$ y $t^{\Delta}$. Reportaron un elevado porcentaje de reforzadores perdidos y frecuencias de respuesta más altas en $t^{\Delta}$ que en $t^{D}$. Estos resultados permitieron a Ribes et al. descartar la modalidad de los estímulos utilizados (visual o auditiva) como una fuente que interfiriera con el desarrollo del control del estímulo.

Serrano, Moreno, Camacho, Aguilar y Carpio (2006) evaluaron los efectos de la dimensión física de los estímulos correlacionados a $t^{\mathrm{D}}$ y $\mathrm{t}^{\mathrm{t}}$ en el desarrollo del control del estímulo. Los estímulos fueron de modalidad auditiva y modalidad visual. Para dos grupos de ratas los estímulos correlacionados a los subciclos $t^{D}$ y $t^{\Delta}$ fueron de la misma dimensión (visual-visual, auditivoauditivo), mientras que en otros dos grupos las modalidades de los estímulos correlacionados a cada subciclo fueron visual-auditiva y auditiva-visual. Serrano et al. reportaron que únicamente en el grupo con estímulos de mo- 
dalidad visual correlacionados a $\mathrm{t}^{\mathrm{D}}$ y $\mathrm{t}^{\Delta}$ se observó una mayor frecuencia de respuesta durante $t^{\Delta}$ que durante $t^{\mathrm{D}}$, así como que la distribución y frecuencia de respuestas durante $t^{\Delta}$ siguió una tendencia a incrementar a medida que finalizaba el ciclo. En el resto de los grupos reportaron bajas tasas de respuesta tanto en $t^{D}$ como en $t^{\Delta}$, en comparación con los sujetos del grupo visual-visual. También reportaron una escasa diferencia entre las tasas de respuesta en $t^{D}$ y $t^{\Delta}$, siendo ligeramente más alta en $t^{D}$. Estos resultados, específicamente los del grupo con estímulos de modalidad visual y los reportados en el presente estudio, son consistentes entre sí y coinciden en que los sujetos obtuvieron un elevado porcentaje de reforzadores programados a diferencia de los trabajos reportados por Ribes et al. (1999; 2000; 2000a; 200b; 2002) y por Ribes y Torres (1996; 1997), en los cuales el porcentaje de reforzadores obtenidos es relativamente bajo o escaso.

Observar mayores tasas de respuesta en $\mathrm{t}^{\Delta}$, en particular en el grupo IEC-3, es un hallazgo consistente con el reportado por Flores et al. (2007) y en la serie de estudios de Ribes et al. (1999; 2000; 2000a; 200b; 2002) y de Ribes y Torres $(1996 ; 1997)$ en cuanto a una ausencia del control del estímulo (i.e. mayores frecuencias de respuesta ante el estímulo correlacionado con no reforzamiento); específicamente en aquellos arreglos experimentales donde se mantiene la presencia de la señal correlacionada a $t^{\Delta}$ y se cancela la señal en $\mathrm{t}^{\mathrm{D}}$ por la emisión de la primera respuesta. La inserción de un intervalo entre ciclos alteró la contigüidad temporal entre la señal correlacionada a $t^{\Delta}$ y la señal correlacionada a $t^{D}$ en el subsecuente ciclo, permitiendo sugerir que la mayor tasa de repuesta observada en $\mathrm{t}^{\Delta}$ puede ser el resultado de un control desarrollado por las propiedades reforzantes del estímulo correlacionado a dicho período.

Los resultados del presente estudio son consistentes con los reportados por Escobar y Bruner (2008) al agregar un intervalo entre los componentes de reforzamiento y extinción, particularmente cuando el intervalo se agregó al final del componente de extinción previo a la ocurrencia de un componente de reforzamiento. Escobar y Bruner explicaron que el mantenimiento de respuestas de observación durante el componente de extinción se debió a las propiedades reforzantes que desarrolla el estímulo correlacionado al componente de extinción como resultado de la eventual contigüidad entre la señal correlacionada al componente de extinción y la señal correlacionada al componente de reforzamiento. En consonancia con lo anterior, la mayor o menor contigüidad de la señal correlacionada a $t^{\Delta}$ con el inicio del siguiente ciclo T modificó sustancialmente la frecuencia y distribución de respuestas durante $t^{\Delta}$.

El hecho de observar tasas de respuesta más bajas en $\mathrm{t}^{D}$ y $\mathrm{t}^{\Delta}$ en los sujetos con un IEC de $30 \mathrm{~s}$ que las observadas en los sujetos con un IEC de $3 \mathrm{~s}$, puede ser interpretado como resultado de una manipulación confundida 
del intervalo entre reforzadores. En el caso de los sujetos con un IEC de 3 $\mathrm{s}$ el intervalo entre reforzadores programado fue de $63 \mathrm{~s}$, mientras que en los sujetos con un IEC de $30 \mathrm{~s}$ el intervalo entre reforzadores fue de $90 \mathrm{~s}$. Lo que esta variación mostraría, en todo caso, es que una condición con un mayor intervalo entre reforzadores contribuye a observar menores tasas de respuesta en $t^{\Delta}$ comparada con una condición con un menor intervalo entre reforzadores, como sería el caso de los sujetos con un IEC de $30 \mathrm{~s}$ comparado con los sujetos con un IEC de $3 \mathrm{~s}$, respectivamente.

Un trabajo reciente que exploró los efectos de la duración del intervalo entre reforzadores sobre la tasa de respuesta en $t^{D}$ y $t^{\Delta}$ fue reportado por Ribes, Montes y Mayoral (2008). Estos autores expusieron a ratas a diferentes duraciones del intervalo entre reforzadores manipulando entre fases la duración del ciclo T (60, 80 y 120 s). De manera general, reportaron que la tasas de respuesta en $t^{D}$ y $t^{\Delta}$ fueron similares entre las diferentes duraciones del ciclo T. Este resultado no es consistente con los reportados en el presente trabajo a pesar de que también se varió la duración del intervalo entre reforzadores; en el experimento citado por medio de la duración del ciclo $\mathrm{T}$, mientras que en el presente estudio por medio de la duración del IEC.

En este sentido, observar tasas de respuesta más altas en $\mathrm{t}^{\mathrm{D}}$ que en $\mathrm{t}^{\Delta}$ en los sujetos con un IEC de mayor duración (30 s) y menores tasas de respuesta en $\mathrm{t}^{\mathrm{D}}$ que en $\mathrm{t}^{\Delta}$ en los sujetos con un IEC de menor duración $(3 \mathrm{~s})$, no puede ser explicado como un efecto de la manipulación confundida de la duración del intervalo entre reforzadores. De ser correcta esta interpretación, Ribes et al. (2008) deberían haber observado tasas de respuesta más altas en $t^{D}$ que en $\mathrm{t}^{\Delta}$ en la condición un ciclo T de mayor duración (120 s) y un efecto inverso en la condición con un menor intervalo entre reforzadores ( $T=60 \mathrm{~s}$ ).

Antes de avanzar y elaborar una explicación concluyente a los presentes resultados, resulta necesario evaluar diferentes aspectos vinculados con la posible función reforzante que desarrolla el estímulo correlacionado a $t^{\Delta}$. Manipulaciones adicionales a la separación temporal entre el estímulo y el reforzador primario son necesarias. En este sentido, es conveniente llevar a cabo manipulaciones bajo las cuales no se altere la secuencia de los ciclos temporales procurando mantener constante el intervalo entre reforzadores, variando la duración del intervalo estímulo-reforzador y con ello la contigüidad de la señal en $t^{\Delta}$ con el siguiente ciclo $\mathrm{T}$. La realización de pruebas de extinción también resulta indispensable para explorar la función reforzante que pudiera estar desarrollando la señal en $t^{\Delta}$. Ello permitiría dar cuenta de la ausencia del control del estímulo en programas temporales, tener una comprensión general del fenómeno, así como de las diferentes funciones que pueden desarrollar los estímulos. 


\section{REFERENCIAS}

Bersh, P. J. (1951). The influence of two variables upon the establishment of a secondary reinforcer for operant responses. Journal of Experimental Psychology, 41, 62-73.

Escobar, R. \& Bruner, C. (2008). Effects of the contiguity between the extinction and the reinforcement components in observing-response procedures. Revista Mexicana de Análisis de la Conducta, 34, 333-347.

Farmer, J. \& Schoenfeld, W. N. (1966). Varying temporal placement of an added stimulus in a fixed-interval schedule. Journal of the Experimental Analysis of Behavior, 9, 369-375.

Ferster, C. B. \& Skinner, B. F. (1957). Schedules of reinforcement. Nueva York: Appleton-Century- Crofts.

Flores, C. \& Mateos, R. (2007). Efectos del mantenimiento de la señal discriminativa y su duración sobre el control del estímulo en programas definidos temporalmente. Trabajo presentado en el xVIII Congreso Mexicano de Análisis de la Conducta. Xalapa, Veracruz, México, noviembre de 2007.

Flores, C., Mateos, R., Villanueva, S. \& Ortiz, R. (2007). Control del estímulo en programas definidos temporalmente: el papel del mantenimiento o cancelación de las señales correlacionada con reforzamiento y extinción. Acta Comportamentalia, 15, 21-33.

Harrison, J. M. (1994). The representative animal. The Behavior Analyst, 17, 207219.

Hearst, E., Besley, S. \& Farthing, G. W. (1970). Inhibition and the stimulus control of operant behavior. Journal of the Experimental Analysis of Behavior, 14, 373-409.

Mackintosh, N. J. (1977). Stimulus control: Attentional factors. En W. K. Honig \& J. E. R. Staddon (Eds.), Handbook of operant behavior (pp. 481-513). New Jersey: Prentice Hall.

Peralta, C., Mateos, R., Villanueva, S. \& Flores, C. (2007). Efectos de la duración del estímulo discriminativo y del estímulo delta en el desarrollo del control del estímulo en programas temporales. Trabajo presentado en el XVIII Congreso Mexicano de Análisis de la Conducta. Xalapa, Veracruz, México, noviembre de 2007.

Ribes, E., Mayoral, A., Torres, C. \& Ibáñez, F. J. (2000). Effects of auditory stimuli correlated with different probabilities of water delivery in a limited-hold temporal schedule. Behavioural Processes, 52, 49-59.

Ribes, E., Montes, E. \& Mayoral, A. (2008). Efectos de la coextensividad del estímulo neutro y distintas longitudes del tiempo discriminativo en un programa definido temporalmente. Acta Comportamentalia, 16, 273-288.

Ribes, E. \& Torres, C. (1996). Efectos de la variación en la probabilidad de reforzamiento correlacionada con dos estímulos neutros en un programa definido temporalmente. Revista Mexicana de Análisis de la Conducta, 22, 41-78.

Ribes, E. \& Torres, C. (1997). Stimulus functions: Lack of discrimination or faluire of generalization? Revista Mexicana de Análisis de la Conducta, 23, 249-274. 
Ribes, E., Torres, C. \& Mayoral, A. (2000a). Señalización no diferencial de distintas probabilidades de entrega de agua en dos subciclos de un programa definido temporalmente. Acta Comportamentalia, 8, 5-21.

Ribes, E., Torres, C. \& Mayoral, A. (2000b). Efectos de la ausencia y presencia de estímulos correlacionados con distintas probabilidades de reforzamiento y con extinción en programas definidos temporalmente. Revista Mexicana de Análisis de la Conducta, 26, 327-354.

Ribes, E., Torres, C. \& Mayoral, A. (2002). Extended exposure to a discriminated, limited-hold temporal schedule does not produce stimulus control. Behavioural Processes, 59, 131-146.

Ribes, E., Torres, C. \& Piña, J. (1999). Comparación de los efectos de la presencia y ausencia de estímulos diferenciales en dos programas temporales con probabilidades variantes igualadas. Acta Comportamentalia, 7, 5-29.

Rilling, M. (1977). Stimulus control and inhibitory processes. En W. K. Honig \& J. E. R. Staddon (Eds.) Handbook of operant behavior (pp. 432-480). New Jersey: Prentice Hall.

Schoenfeld, W. N. \& Cole, B. K. (1972). Stimulus schedules: the T-t system. Nueva York: Harper.

Schoenfeld, W. N., Cumming, W. W. \& Hearst, E. (1956). On the classification of reinforcement schedules. Proceedings of the National Academy of Sciences. 42, 563570.

Serrano, M., Moreno, S., Camacho, I., Aguilar, F. \& Carpio, C. (2006). Dimensión física de las señales agregadas en programas definidos temporalmente. Revista Mexicana de Análisis de la Conducta, 32, 13-25.

Terrace, H. S. (1966). Stimulus control. En W. K. Honig (Ed.) Operant behavior: Areas of research and application (pp. 271-344). Nueva York: Appleton Century Crofts.

Weissman, A. (1961). Impairment of performance when a discriminative stimulus is correlated with a reinforcement contingency. Journal of the Experimental Analysis of Behavior, 4, 365-369.

Weissman, A. (1965). Behavior effects of pairing an SD with a decreasing limited-hold reinforcement schedule. Journal of the Experimental Analysis of Behavior, 6, 265268. 